\title{
Gravidade do déficit neurológico e incidência de infecções hospitalares em pacientes idosos com acidente vascular cerebral agudo
}

\author{
Severity of neurological deficit and incidence of nosocomial infections \\ in elderly patients with acute stroke
}

\author{
Lucieni Oliveira Conterno ${ }^{1} \bowtie$, Rodrigo Wanderley Neves Barbosa ${ }^{1}$, Caroline Martins Rego ${ }^{1}$, \\ Carlos Rodrigues da Silva Filho'
}

${ }^{1}$ Disciplina de Infectologia da Faculdade de Medicina de Marília. Marília, SP.

2 Disciplina de Clínica Médica da Faculdade de Medicina de Marília. Marília, SP.

\section{RESUMO}

Objetivos: Avaliar a associação entre gravidade do déficit neurológico/grau de incapacidade funcional e incidência de infecções hospitalares, tempo de permanência hospitalar e mortalidade, em pacientes idosos com acidente vascular cerebral agudo.

Métodos: Um estudo de coorte prospectivo avaliou pacientes idosos com diagnóstico de acidente vascular cerebral agudo, internados no Hospital de Clínicas da Faculdade de Medicina de Marília, em Marília, SP. Os critérios de inclusão foram idade igual ou superior a 60 anos e diagnóstico de acidente vascular cerebral agudo. Os pacientes foram agrupados de acordo com a gravidade do acidente vascular cerebral pela National Institute of Health Stroke Scale e o grau de dependência pela escala modificada de Rankin. Todos os pacientes foram acompanhados desde o momento da internação até a alta hospitalar ou óbito.

Resultados: Foram estudados 113 pacientes com média de idade de 70,8 anos, sendo $63(55,7 \%)$ homens. As principais doenças de base relacionadas foram hipertensão arterial $(77,9 \%)$ e diabetes mellitus tipo $2(30,1 \%)$. Setenta e oito $(69 \%)$ pacientes foram acometidos pela primeira vez e $86(76,1 \%)$ apresentaram acidente vascular cerebral isquêmico. Tiveram infecção hospitalar 24 pacientes $(21,2 \%)$ sendo pneumonia a mais frequente $(13,5 \%)$. O tempo médio de permanência hospitalar dos pacientes com infecção hospitalar foi maior, quando comparados àqueles sem infecção hospitalar $(16,2 \pm 16,2$ dias e 33,2 222,9 dias, $\mathrm{p}<0,001)$. Observou-se relação entre escore de 16 a 42 pontos, na National Institute of Health Stroke Scale, e a ocorrência de infecção hospitalar (rico relativo 4,4; intervalo de confiança 95\% 1,8 a 11,0). A mortalidade intra-hospitalar foi de $15,9 \%$, sendo maior entre pacientes considerados muito graves à admissão, quando comparados com aqueles com gravidade moderada a leve pela National Institute of Health Stroke Scale, $(34,6 \%$ e 1,6\% respectivamente, p<0,001) e também entre aqueles que desenvolveram infecção hospitalar quando comparados aos que não apresentaram esta complicação $(37,5 \%$ e $10,1 \%$ respectivamente, $\mathrm{p}=0,003)$.

Conclusões: As infecções hospitalares foram complicações importantes entre os pacientes idosos com acidente vascular cerebral agudo e estiveram associadas com maior gravidade do déficit neurológico, maior grau de dependência, tempo de hospitalização mais prolongado e taxa de mortalidade mais alta.

DESCRITORES: acidente vascular cerebral; infecção hospitalar; epidemiologia; mortalidade; idosos.

\section{ABSTRACT}

Aims: To evaluate the association between severity of neurological deficit/degree of functional disability and incidence of nosocomial infections, length of hospital stay, and mortality, in elderly patients with acute stroke.

Methods: A prospective cohort study evaluated elderly patients with acute stroke, hospitalized at the Hospital of the Marilia Medical School, in Marilia, SP, Brazil. Inclusion criteria were patients aged 60 years or more and diagnosed with acute stroke. Patients were stratified according to the severity of the stroke by the National Institutes of Health Stroke Scale and the degree of dependence by the modified Rankin scale. All patients were followed from the time of admission to discharge or death.

Results: We studied 113 patients with a mean age of 70.8 years, $63(55.7 \%)$ men. The main underlying diseases were hypertension $(77.9 \%)$ and type 2 diabetes mellitus (30.1\%). Seventy-eight (69\%) patients were affected for the first time and $86(76.1 \%)$ had ischemic stroke. Nosocomial infection occurred in 24 patients $(21.2 \%)$ and pneumonia was the most frequent $(13.5 \%)$. The average length of hospital stay among patients with nosocomial infection was higher when compared to those without nosocomial infection (16.2 \pm 16.2 days and $33.2 \pm 22.9$ days, $p<0.001)$. There was association between score $16-42$ points on the National Institute of Health Stroke Scale and the occurrence of nosocomial infection (risk ratio $4.4 ; 95 \%$ confidence interval 1.8 to 11.0 ). In-hospital mortality was $15.9 \%$, higher among patients considered severe at admission compared to those with moderate to mild severity by the National Institute of Health Stroke Scale (34.6\% and $1.6 \%$ respectively, $\mathrm{p}<0.001)$ and also among those who developed nosocomial infection when compared to those without this complication $(37.5 \%$ and $10.1 \%$ respectively, $\mathrm{p}=0.003$ ).

Conclusions: Nosocomial infections were major complications among elderly patients with acute stroke and were associated with severity of neurological deficit, higher degree of dependence, longer hospital stay and higher mortality rate.

KEY WORDS: stroke; cross infection; epidemiology; mortality; elderly. 
Abreviaturas: AVC, acidente vascular cerebral; IH, infecção hospitalar; NIHSS, National Institute of Health Stroke Scale; UTI, unidade de tratamento intensivo.

\section{INTRODUÇÃO}

As doenças crônicas não transmissíveis representam as principais causas de internação em pessoas com mais de 60 anos de idade, com destaque para as doenças cardiovasculares. Dentro do grupo das doenças cardiovasculares, o acidente vascular cerebral (AVC) tornou-se a segunda maior causa de mortalidade e a principal causa de incapacidade no mundo [1], embora apresente uma tendência de queda de mortalidade no Brasil, devido ao controle de seus fatores de risco [2].

Segundo a Organização Mundial da Saúde, o AVC caracteriza-se por sinais clínicos de distúrbios focais e/ou globais da função cerebral, de desenvolvimento rápido e sintomas com duração igual ou superior a 24 horas, de origem vascular, que provoca alterações nos planos cognitivo e sensório-motor, de acordo com a área e a extensão da lesão [3].

Os instrumentos National Institute of Health Stroke Scale (NIHSS) e Escala Rankin Modificada são úteis para avaliar o grau de déficit neurológico e a incapacidade funcional associada ao AVC, tanto no atendimento clinico como em pesquisa [4-6]. A escala NIHSS é um instrumento de uso sistemático para uma avaliação quantitativa do déficit neurológico relacionado com o AVC, pois serve para documentar e monitorar o estado neurológico do paciente e prever a extensão e a gravidade do AVC. Além disso, representa uma ferramenta importante para determinação do prognóstico e orientação do tratamento adequado, garantindo uma linguagem universal para troca de informações entre os profissionais de saúde. A NIHSS é composta por 11 categorias, com 15 itens do exame neurológico, pontuados de zero a quatro, dependendo do item, o que leva a um escore geral. Os 15 itens da escala NIHSS avaliam o efeito do AVC agudo e definem gravidade. Quanto maior o escore, mais sérias as consequências do agravo [4]. A Escala de Rankin Modificada, por sua vez, é utilizada para avaliar o estado funcional e o grau de incapacidade para a realização das atividades da vida diária. É pontuada de zero a seis pontos, onde o zero define a ausência de sintomas e o seis a morte, passando pelos escores intermediários de progressiva gravidade [5]. Essas escalas, já validadas no Brasil, apresentam entre si uma forte correlação positiva, e quanto maiores seus escores, maiores a gravidade e o potencial de morte $[6,7]$.

Pacientes com AVC têm alto risco de apresentar várias complicações durante a evolução do quadro agudo, que aumentam a probabilidade de sequelas ou mesmo da evolução para óbito. As complicações clínicas após AVC interrompem o processo de reabilitação das sequelas, comprometendo a melhora funcional, resultando em maior morbidade e impacto na qualidade de vida [8]. As complicações infecciosas adquiridas pós-AVC podem determinar pior evolução dos pacientes, independente da estratégia de tratamento utilizada [9-11].

Os objetivos deste estudo foram avaliar a associação entre gravidade do déficit neurológico/grau de incapacidade funcional em idosos com AVC agudo, e a incidência de infecções hospitalares (IH), tempo de permanência hospitalar e mortalidade.

\section{MÉTODOS}

Foi realizado um estudo de coorte prospectivo, que incluiu pacientes idosos com diagnóstico de AVC internados consecutivamente, no Hospital de Clínicas da Faculdade de Medicina de Marília, no período de 05 de janeiro de 2014 a 01 de dezembro de 2015. Os critérios de inclusão foram: idade igual ou superior a 60 anos e diagnóstico de AVC agudo. Foram excluídos os pacientes com sequelas de AVC, internados por complicações clínicas. Os pacientes foram agrupados de acordo com a gravidade pela escala NIHSS e com o estado funcional pela escala de Rankin Modificada, aplicadas à admissão. Todos foram acompanhados ao longo da internação para documentar a ocorrência ou não de IH, até alta hospitalar ou óbito (desfecho). Fizeram parte desse estudo 113 pacientes.

O diagnóstico de AVC baseou-se nos critérios preconizados pela American Heart Association e American Stroke Association [12]. Os critérios para o diagnóstico das IH foram os preconizado pela Secretaria de Estado da Saúde de São Paulo [13] que se baseiam nas definições do Centers for Disease Control and Prevention dos Estados Unidos [14].

Realizou-se análise descritiva e comparativa dos dados. As proporções foram comparadas pelo teste de Qui-quadrado de Pearson, e as médias pelo teste $t$ de Student. Foi realizada análise de correlação através do teste de Pearson comparando-se os valores obtidos na escala NIHSS e na escala Rankin. O projeto foi submetido à apreciação do Comitê de Ética em Pesquisa da Faculdade de Medicina de Marília e aprovado conforme o parecer consubstanciado de no 204.922. 


\section{RESULTADOS}

Foram estudados 113 pacientes com média de idade de 70,8 anos, sendo 63 (55,7\%) homens, 62 $(54,9 \%)$ casados e $79(69,9 \%)$ inativos.

As comorbidades mais frequentemente identificadas foram hipertensão arterial, em $88(77,9 \%)$ e diabetes mellitus em 34 (30,1\%) pacientes. Setenta e oito pacientes $(69,0 \%)$ foram acometidos pela primeira vez, enquanto $35(31,0 \%)$ já haviam apresentado pelo menos um episódio anterior. Em $86(76,1 \%)$ casos, o AVC foi classificado como isquêmico. Em relação ao território acometido, em $77(68,1 \%)$ foi o território da artéria cerebral média, seguidos por $21(18,6 \%)$ com acometimento do território da artéria cerebral anterior (Tabela 1).

A gravidade do AVC à admissão, baseando-se na Escala NIHSS, foi em média de 17,8 pontos, sendo que 52 pacientes $(46,0 \%)$ pontuaram entre $16-42$, que indica maior gravidade. Na avaliação pela Escala Rankin, à admissão, 61 (54,0\%) pacientes apresentavam dependência grave, com pontuação entre 4-5. Comparando os valores obtidos nas escalas NIHSS e Rankin, pelo coeficiente de correlação de Pearson, encontrou-se relação positiva e significante $(\mathrm{r}=0,747$, $\mathrm{p}<0,001)$. Os dados sobre as características do AVC são mostrados na Tabela 1.

Tabela 1. Características e classificação do episódio de acidente vascular cerebral entre 113 pacientes idosos internados no complexo FAMEMA - Hospital de Clínicas da Faculdade de Medicina de Marília. Marília, SP, 2014-2015.

\begin{tabular}{|c|c|c|}
\hline Características e classificação do AVC & $\mathbf{n}$ & $\%$ \\
\hline $\begin{array}{l}\text { Episódio } \\
\text { Primeiro episódio } \\
\text { Recorrência }\end{array}$ & $\begin{array}{l}78 \\
35\end{array}$ & $\begin{array}{l}69 \\
31\end{array}$ \\
\hline $\begin{array}{l}\text { Tipo } \\
\text { Isquêmico } \\
\text { Hemorrágico }\end{array}$ & $\begin{array}{l}86 \\
27\end{array}$ & $\begin{array}{l}76,1 \\
23,9\end{array}$ \\
\hline $\begin{array}{l}\text { Localização } \\
\text { Cerebral anterior } \\
\text { Cerebral média } \\
\text { Cerebral posterior } \\
\text { Cerebelar superior } \\
\text { Cerebelar anterior inferior } \\
\text { Cerebelar posterior inferior }\end{array}$ & $\begin{array}{c}21 \\
77 \\
8 \\
4 \\
2 \\
1\end{array}$ & $\begin{array}{c}18,6 \\
68,1 \\
7,1 \\
3,5 \\
1,8 \\
0,9\end{array}$ \\
\hline $\begin{array}{l}\text { NIHSS à admissão (média } \pm \text { DP: } 17,8 \pm 14,9 \text { ) } \\
\text { Pontuação } \\
0-5 \\
6-15 \\
16-42\end{array}$ & $\begin{array}{l}33 \\
28 \\
52\end{array}$ & $\begin{array}{l}29,2 \\
24,8 \\
46,0\end{array}$ \\
\hline $\begin{array}{l}\text { Rankin à admissão } \\
\text { Sem dependência (0-1) } \\
\text { Dependência moderada (2-3) } \\
\text { Dependência grave (4-5) }\end{array}$ & $\begin{array}{l}20 \\
32 \\
61\end{array}$ & $\begin{array}{l}17,7 \\
28,3 \\
54,0\end{array}$ \\
\hline $\begin{array}{l}\text { Internação em UTI } \\
\text { Não } \\
\text { Sim }\end{array}$ & $\begin{array}{l}96 \\
17 \\
\end{array}$ & $\begin{array}{l}85,0 \\
15,0\end{array}$ \\
\hline
\end{tabular}

DP, desvio padrão; AVC, acidente vascular cerebral; NIHSS, National Institutes of Health Stroke Scale; Rankin, escala de Rankin Modificada, para avaliação do estado funcional dos pacientes vítimas de AVC; UTI, unidade de tratamento intensivo.
O tempo médio de hospitalização foi de $16,2 \pm 16,2$ dias. Dezessete pacientes $(14,1 \%)$ foram internados em Unidade de Tratamento Intensivo (UTI) e a média dos dias de internação foi de $17 \pm 13,9$ dias. Em relação aos procedimentos invasivos de suporte, a sondagem vesical de demora foi realizada em 41 (36,3\%) pacientes, por um tempo médio de uso de 5,8 $\pm 11,7$ dias; o acesso venoso periférico em 110 (97,3\%) pacientes, com tempo médio de manutenção do acesso


em $46(40,7 \%)$ pacientes, por um tempo médio de $8,7 \pm 16,2$ dias.

Desenvolveram IH 24 pacientes $(21,2 \%)$, sendo dezoito casos de pneumonia $(15,9 \%)$, cinco de infecção urinária $(4,4 \%)$ e quatro de infecção da corrente sanguínea (3,5\%). Antimicrobianos foram utilizados por 28 pacientes $(24,8 \%)$.

Observou-se relação entre escore de 16 a 42 pontos na escala NIHSS e a ocorrência de IH: risco relativo (RR) 4,4; IC 95\%; 1,8-11,0. Pacientes classificados como portadores de incapacidade grave pela escala Rankin, com valores entre 4 e 5 , também tiveram mais IH quando comparados àqueles com escores entre 0 e 3 (respectivamente $36,1 \%$ e 3,8\%; RR 9,4 IC95\% 2,3-38,0) (Tabela 2).

A incidência acumulada de IH foi maior entre aqueles pacientes que usaram por maior tempo a sonda vesical de demora e a sonda nasogástrica. O tempo médio de permanência hospitalar dos pacientes com AVC e IH foi maior quando comparados àqueles sem IH: respectivamente $16,2 \pm 16,2$ dias e $33,2 \pm 22,9$ dias, diferença média de 8,9 dias $(\mathrm{p}<0,001)$. Dos 113 pacientes acompanhados, 88 pacientes $(77,9 \%)$ receberam alta hospitalar, $18(15,9 \%)$ evoluíram para óbito e sete $(6,2 \%)$ foram transferidos para outro hospital.

Um escore na escala NIHSS entre 16-42 à admissão, considerado grave, associou-se à maior taxa de mortalidade $(34,6 \%)$, em relação à taxa $(1,6 \%)$ observada nos pacientes com escore entre 0 e 15 (gravidade leve a moderada), (RR 1,5; IC95\% 1,3-1,9; $\mathrm{p}<0,001$,). Da mesma forma, entre os pacientes com valores entre quatro e cinco, com maior incapacidade conforme a escala modificada de Rankin, 27,9\% evoluíram para óbito, enquanto a mortalidade entre os pacientes com valores entre 1 e 3 , ou seja, incapacidade leve a moderada, foi de 1,9\% (RR 1,4 IC95\% 1,2-1,6, $\mathrm{p}<0,001)$. Não houve associação entre mortalidade e localização do AVC.

Dos 24 pacientes com IH, nove (37,5\%) evoluíram para óbito, enquanto dos 89 pacientes sem $\mathrm{IH}$, nove (10,1\%) foram a óbito (RR 3,7 IC95\% 1,6-8,3, $\mathrm{p}=0,003)$ (Tabela 3). 
Tabela 2. Comparação das características clínicas e epidemiológicas entre 113 pacientes idosos com acidente vascular cerebral, com e sem infecção hospitalar, internados no complexo FAMEMA - Hospital de Clínicas da Faculdade de Medicina de Marília. Marília, SP, 2014-2015.

\begin{tabular}{|c|c|c|c|c|c|}
\hline Características dos idosos com AVC & $\begin{array}{c}\text { Com infecção hospitalar } \\
n(\%)\end{array}$ & $\begin{array}{c}\text { Sem infecção hospitalar } \\
n(\%)\end{array}$ & $\mathbf{R R}$ & IC $95 \%$ & $\mathbf{p}^{+}$ \\
\hline $\begin{array}{l}\text { Sexo } \\
\quad \text { Homens } \\
\text { Mulheres }\end{array}$ & $\begin{array}{r}15(23,8) \\
9(18,0)\end{array}$ & $\begin{array}{l}48(76,2) \\
41(82,0)\end{array}$ & 1,3 & $0,6-2,7$ & 0,495 \\
\hline $\begin{array}{l}\text { Idade } \\
\quad \geq 80 \text { anos } \\
\quad<80 \text { anos }\end{array}$ & $\begin{array}{r}5(31,3) \\
19(18,0)\end{array}$ & $\begin{array}{l}48(76,2) \\
41(82,0)\end{array}$ & 1,2 & $0,8-1,6$ & 0,326 \\
\hline $\begin{array}{l}\text { Restrito ao leito } \\
\text { Sim } \\
\text { Não }\end{array}$ & $\begin{array}{c}20(32,3) \\
4(7,8)\end{array}$ & $\begin{array}{l}42(67,7) \\
47(92,2)\end{array}$ & 4,1 & $1,5-11,2$ & 0,002 \\
\hline $\begin{array}{l}\text { Tipo do AVC } \\
\text { Hemorrágico } \\
\text { Isquêmico }\end{array}$ & $\begin{array}{l}11(40,7) \\
13(15,1)\end{array}$ & $\begin{array}{l}16(59,3) \\
73(84,9)\end{array}$ & 0,6 & $0,5-0,9$ & 0,007 \\
\hline $\begin{array}{l}\text { Episódio de AVC } \\
\text { Recorrência } \\
\text { Primeiro }\end{array}$ & $\begin{array}{r}9(25,7) \\
15(19,2)\end{array}$ & $\begin{array}{l}26(74,3) \\
63(80,8)\end{array}$ & 1,1 & $0,9-1,4$ & 0,462 \\
\hline $\begin{array}{l}\text { Localização } \\
\text { Cerebral média } \\
\text { Outras localizações }\end{array}$ & $\begin{array}{r}17(22,1) \\
7(12,5)\end{array}$ & $\begin{array}{l}60(77,9) \\
29(71,4)\end{array}$ & 1,1 & $0,5-2,5$ & 0,749 \\
\hline $\begin{array}{c}\text { NIHSS } \\
16-42 \\
0-15\end{array}$ & $\begin{aligned} 19 & (36,5) \\
5 & (8,2)\end{aligned}$ & $\begin{array}{l}33(63,5) \\
56(91,8)\end{array}$ & 4,4 & $1,8-11,0$ & $<0,001$ \\
\hline $\begin{array}{c}\text { Rankin } \\
4-5 \\
0-3\end{array}$ & $\begin{aligned} 22 & (36,1) \\
2 & (3,8)\end{aligned}$ & $\begin{array}{l}39(63,9) \\
50(96,2)\end{array}$ & 9,4 & $2,3-38,0$ & $<0,001$ \\
\hline $\begin{array}{l}\text { Internação em UTI } \\
\text { Não } \\
\text { Sim }\end{array}$ & $\begin{array}{r}15(15,6) \\
9(52,9)\end{array}$ & $\begin{array}{l}1(84,4) \\
8(47,1)\end{array}$ & 0,3 & $0,1-0,5$ & 0,002 \\
\hline
\end{tabular}

AVC, acidente vascular cerebral; RR, risco relativo; IC, intervalo de confiança; NIHSS, National Institute of Health Stroke Scale; Rankin, escala de Rankin Modificada; UTI, unidade de tratamento intensivo. + Teste qui-quadrado.

Tabela 3. Comparação das características clínicas e epidemiológicas conforme os desfechos óbito ou sobrevida, em 113 pacientes idosos com acidente vascular cerebral internados no complexo FAMEMA - Hospital de Clínicas da Faculdade de Medicina de Marília. Marília, SP, 2014-2015.

\begin{tabular}{|c|c|c|c|c|c|}
\hline Características dos idosos com AVC & $\begin{array}{l}\text { Óbito } \\
\text { n (\%) }\end{array}$ & $\begin{array}{c}\text { Sobrevida } \\
\text { n (\%) }\end{array}$ & $\mathbf{R R}$ & IC95\% & $\mathbf{p}^{+}$ \\
\hline $\begin{array}{l}\text { Sexo } \\
\quad \text { Homens } \\
\text { Mulheres }\end{array}$ & $\begin{array}{c}8(16,0) \\
10(15,9)\end{array}$ & $\begin{array}{l}42(84,0) \\
53(84,1)\end{array}$ & 1,0 & $0,8-1,2$ & 1,000 \\
\hline $\begin{array}{l}\text { Idade } \\
\quad \geq 80 \text { anos } \\
\quad<80 \text { anos }\end{array}$ & $\begin{array}{c}3(18,8) \\
15(15,5)\end{array}$ & $\begin{array}{l}13(81,3) \\
82(84,5)\end{array}$ & 1,3 & $0,4-3,7$ & 0,718 \\
\hline $\begin{array}{l}\text { Restrito ao leito } \\
\text { Não } \\
\text { Sim }\end{array}$ & $\begin{array}{c}0(0,0) \\
18(29,0)\end{array}$ & $\begin{array}{l}51(100,0) \\
44(71,0)\end{array}$ & 0,07 & $0,6-0,8$ & $<0,001$ \\
\hline $\begin{array}{l}\text { Tipo do AVC } \\
\text { Isquêmico } \\
\text { Hemorrágico }\end{array}$ & $\begin{array}{r}14(16,3) \\
4(14,8)\end{array}$ & $\begin{array}{l}72(83,7) \\
23(85,2)\end{array}$ & 1,0 & $0,8-1,2$ & 1,000 \\
\hline $\begin{array}{l}\text { Episódio de AVC } \\
\text { Recorrência } \\
\text { Primeiro }\end{array}$ & $\begin{array}{r}10(28,6) \\
8(10,3)\end{array}$ & $\begin{array}{l}25(71,4) \\
70(89,7)\end{array}$ & 1,3 & $1,0-1,6$ & 0,017 \\
\hline $\begin{array}{l}\text { Localização da lesão } \\
\text { Cerebral média } \\
\text { Outras localizações }\end{array}$ & $\begin{array}{r}11(14,3) \\
7(19,4)\end{array}$ & $\begin{array}{l}66(85,7) \\
29(80,6)\end{array}$ & 0,73 & $0,3-1,7$ & 0,485 \\
\hline $\begin{array}{l}\text { NIHSS } \\
16-42 \\
0-15\end{array}$ & $\begin{array}{c}17(34,6) \\
1(1,6)\end{array}$ & $\begin{array}{l}34(65,4) \\
61(98,4)\end{array}$ & 1,5 & $1,3-1,9$ & $<0,001$ \\
\hline $\begin{array}{c}\text { Rankin } \\
4-5 \\
0-3\end{array}$ & $\begin{array}{c}17(27,9) \\
1(1,9)\end{array}$ & $\begin{array}{l}44(72,1) \\
51(98,1)\end{array}$ & 1,4 & $1,2-1,6$ & $<0,001$ \\
\hline $\begin{array}{l}\text { Internação em UTI } \\
\text { Não } \\
\text { Sim }\end{array}$ & $\begin{array}{r}14(14,6) \\
4(23,5)\end{array}$ & $\begin{array}{l}82(85,4) \\
13(76,5)\end{array}$ & 0,6 & $0,2-1,6$ & 0,469 \\
\hline $\begin{array}{l}\text { Infecção hospitalar } \\
\text { Sim } \\
\text { Não }\end{array}$ & $\begin{array}{l}9(37,5) \\
9(10,1)\end{array}$ & $\begin{array}{l}15(62,5) \\
80(89,9)\end{array}$ & 3,7 & $1,6-8,3$ & 0,003 \\
\hline
\end{tabular}

RR, risco relativo; IC, intervalo de confiança; AVC, acidente vascular cerebral; NIHSS, National Institute of Health Stroke Scale; Rankin, escala de Rankin Modificada; UTI, unidade de tratamento intensivo. † Teste qui-quadrado 


\section{DISCUSSÃO}

O objetivo desse estudo foi avaliar a relação entre a gravidade do AVC e incapacidade funcional com a incidência de infecção hospitalar e mortalidade, em pacientes idosos, e internados com diagnóstico de AVC, em um hospital geral de ensino. Sabe-se que a incidência do AVC aumenta a partir dos 60 anos e dobra a cada década, que o diabetes causa lesão endotelial aterogênica direta e interage com outros fatores de risco como alterações lipídicas e hipertensão, sendo este o principal fator de risco descrito e potencialmente modificável [15].

Semelhante a outros estudos [16,17], o AVC foi mais frequente na população masculina, associado à hipertensão arterial sistêmica, seguido pelo diabetes. $\mathrm{Ng}$ et al. [17], no estudo realizado em Boston, incluindo 2.213 pacientes, também identificaram a associação dos fatores de risco hipertensão arterial sistêmica e diabetes, sendo que $58,7 \%$ dos pacientes apresentavam dois ou mais fatores de risco, o que também ocorreu em $51,9 \%$ dos pacientes deste estudo.

O escore médio elevado encontrado na escala NIHSS é compatível com outros estudos em hospitais de referência [9,18-20], que recebem pacientes de maior gravidade, como ocorre com o complexo FAMEMA, mesmo não tendo unidade específica de tratamento de agravos cerebrovasculares. Os instrumentos utilizados, NIHSS e a Escala de Rankin, apresentam adequadas propriedades avaliativas e psicométricas na versão para a língua portuguesa [6], assim como há associação dos escores desses instrumentos com a gravidade do dano neurológico central, independente do tipo de AVC [5].

Ao avaliarmos o grau de dependência pela Escala de Rankin Modificada, verificamos que mais da metade dos pacientes apresentavam incapacidade moderada a grave e incapacidade grave, havendo uma concordância entre as duas escalas, provavelmente pelo número de casos graves nesta casuística.

A taxa de IH encontrada no nosso estudo foi elevada, porém menor que a descrita na revisão realizada por Westendorp [21], que avaliou 87 estudos envolvendo 137.817 pacientes e relatou taxas de $24 \%$ a $36 \%$.

As infecções representam a terceira complicação mais comum pós-AVC, sendo suplantadas apenas por quedas e úlceras por pressão. As taxas podem variar de acordo com as características da população estudada e do local de atendimento. Entre as infecções, as mais frequentemente descritas são as de origem respiratória, variando de $7,5 \%$ a $19,9 \%$, e a infecção urinária, com prevalência entre 4,4\% e 10\% [22-25].
Há possíveis explicações para a frequente ocorrência de infecções respiratórias nos pacientes incluídos neste estudo, além da gravidade do AVC. Por ser uma coorte de idosos poderia haver fatores de risco associados, como distúrbios prévios de motilidade esofagiana e deglutição, que demandam a utilização de sonda nasogástrica na fase aguda. Além disso, a inexistência de uma unidade especializada em tratamento de AVC e a insuficiência de leitos de UTI podem determinar que pacientes graves com suporte ventilatório invasivo sejam tratados em enfermarias, com todos os riscos daí advindos.

A relação entre AVC e infecção é complexa e bidirecional. Tanto infecções podem precipitar o AVC [27] como podem ocorrer após o agravo vascular cerebral [22]. Além do estado de imobilidade e restrição física que o AVC moderado a severo frequentemente impõe, há necessidade de hospitalização dos casos graves, por vezes prolongada, inserção de múltiplos cateteres e sondas e eventual necessidade de suporte respiratório invasivo e internação em UTI [28]. Sabe-se que a agressão vascular aguda ao cérebro provoca uma síndrome de imunodeficiência induzida, caracterizada por perda de linfócitos por apoptose, alterações nas funções dos linfócitos T-helper 1 e 2 e na produção de citocinas, diminuição do número e da função de mastócitos e deficiência de interferon, que de forma combinada, elevam a susceptibilidade do organismo às infecções [29]. Além dessas condições, a desnutrição e o próprio processo de envelhecimento estão associados à diminuição dos mecanismos de defesa do organismo e ao aumento da suscetibilidade às infecções [30].

No presente estudo encontramos maior risco de infecção entre os pacientes restritos ao leito e entre aqueles com maior déficit neurológico e incapacidade, evidenciados pela escala NIHSS e de Rankin. Da mesma forma, estudo realizado por Wartenberg et al. [29], que avaliou 96 pacientes com diagnóstico de AVC, a pontuação NIHSS de 13,3 $\pm 0,9$ associou-se com maior risco de infecção quando comparada a menores escores. No estudo realizado por Sellars et al. [24] também foi observado que, quanto maior a pontuação na escala NIHSS, maior o risco de ocorrer infecção. Por exemplo, os pacientes que tiveram mais de seis pontos apresentaram maior chance de infecção (OR 12,9 IC95\% 6,8 a 24,2) [24].

Pacientes com recorrência de AVC apresentam maior risco de infecção e maior mortalidade quando comparados a pacientes que sofreram o primeiro episódio. Certamente concorrem para este desfecho as consequências do envelhecimento, a devastação 
orgânica causada por múltiplas agressões, a síndrome de imobilidade e perda de independência e a desnutrição decorrente desse estado [31].

Disfagia secundária ao agravo vascular está presente em mais de 50\% dos pacientes com AVC [34]. A utilização de sonda nasogástrica e a imobilidade no leito aumentam de forma importante o risco de aspiração, sendo considerados preditivos da ocorrência de pneumonia, assim como de maior mortalidade $[25,26,33]$, como se observou no presente estudo.

Os pacientes que desenvolveram IH tiveram permanência hospitalar média maior que os pacientes sem IH. Um dos grandes impactos das IH, tanto para o paciente como para os serviços de saúde, é o aumento do tempo de permanência hospitalar com consequente aumento da morbidade e dos custos. Novamente essa associação parece ser bidirecional, posto que tanto a longa permanência, por qualquer causa, predispõe à IH como essas levam a um maior tempo de permanência [35]. O estudo de Wartenberg [29] relatou resultados semelhantes aos nossos. O tempo médio de permanência de pacientes com IH naquele estudo foi de 17,3 $\pm 1,2$ dias, sendo que os pacientes sem IH

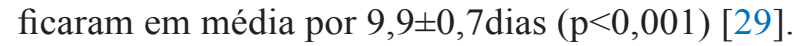

Em geral, a mortalidade entre pacientes com IH varia de $10,9 \%$ a $24,7 \%$ [28], e a ocorrência de IH em pacientes pós-AVC pode representar um aumento de até oito vezes no risco de morte [36]. Comparando a evolução dos pacientes, observamos que aqueles que desenvolveram IH tiveram taxas mais elevadas de óbito quando comparadas com pacientes sem IH. Diversos estudos têm demonstrado este mesmo impacto negativo das infecções na evolução dos pacientes com AVC [25,29,31].
A internação em UTI não se associou com mortalidade em nosso estudo. Talvez isto se deva ao fato de em nossa realidade, a gravidade do caso não seja o critério determinante de internação em UTI, mas sim a disponibilidade ou não de leitos, os quais são muito escassos. Em decorrência disso é cada vez mais frequente pacientes graves internados em enfermaria, não sendo assim possível, considerar a internação em UTI como um marcador de gravidade em todos os casos.

Uma limitação deste estudo foi incluir apenas pacientes idosos, já que a idade avançada é considerada uma variável importante para maior gravidade e pior evolução dos pacientes com AVC. Por outro lado, acreditamos que o presente estudo oferece uma visão de como se encontra a assistência aos pacientes idosos com AVC em um hospital geral de ensino, que atende exclusivamente pacientes do Sistema Único de Saúde, não possui unidade especializada em tratamento de pacientes com AVC, ou número de vagas na UTI que permitam receber todos os pacientes mais graves.

Pode-se concluir que as IH foram complicações importantes entre os pacientes idosos com AVC agudo, e estiveram associadas com maior gravidade do déficit neurológico, maior grau de dependência, tempo de hospitalização mais prolongado e taxa de mortalidade mais alta. É necessário, pois, implementar, dentre tantas outras demandas igualmente relevantes, como a estruturação de unidade especializada de tratamento de pacientes com AVC, medidas especificamente dirigidas à população idosa internada com esse diagnóstico, que visem à prevenção e controle das $\mathrm{IH}$, particularmente as respiratórias, pois estas representam uma grande ameaça à segurança do paciente já fragilizado e um enorme custo para o sistema de saúde.

\section{NOTAS}

Apoio financeiro

Os autores agradecem à Fundação de Amparo a Pesquisa do Estado de São Paulo, pelo apoio. Processo FAPESP № 2013/15895-3.

Declaração de conflitos de interesse

Os autores declaram não haver conflitos de interesse relevantes ao conteúdo deste estudo.

\section{REFERÊNCIAS}

1. Feigin VL, Lawes CM, Bennett DA, Barker-Collo SL, Parag V. Worldwide stroke incidence and early case fatality reported in 56 populationbased studies: a systematic review. Lancet Neurol. 2009;(4):355-69. http://dx.doi.org/10.1016/S1474-4422(09)70025-0

2. Garritano CR, Luz PM, Pires MLE, Barbosa MTS, Batista KM. Análise da tendência da mortalidade por acidente vascular cerebral no Brasil no século XXI. Arq Bras Cardiol. 2012;98(6):519-27. http://dx.doi.org/10.1590/S0066-782X2012005000041

3. World Health Organization. Stroke, cerebrovascular accident [Internet]. Geneva: WHO; 2014. [cited 2014 Sept 20]. Available from: http:// www.who.int/topics/cerebrovascular_accident/en/ 
4. Lyden P, Lu M, Jackson C, Marler J, Kothari R, Brott T, Justin Zivin, NINDS tPA Stroke Trial Investigators. Underlying structure of the National Institutes of Health Stroke Scale: results of a factor analysis. Stroke. 1999;30(11):2347-54. http://dx.doi.org/10.1161/01. STR.30.11.2347

5. Swieten JC, Koudstaal PJ, Visser HJA, Schouten, Gijn J. Interobserver agreement for the assessment of handicap in stroke patients. Stroke. 1988;19(5):604-7. http://dx.doi.org/10.1161/01.STR.19.5.604

6. Caneda MAG, Fernandes JG, Almeida AC, Mugno FE. Confiabilidade de escalas de comprometimento neurológico em pacientes com acidente vascular cerebral. Arq Neuropsiquiatr. 2006;64(3A):690-7. http://dx.doi.org/10.1590/S0004-282X2006000400034

7. Cincura C, Pontes-Neto OM, Neville IS, Mendes HF, Menezes DF, Mariano DC, Pereira IF, Teixeira LA, Jesus PAP, Queiroz DCL, Pereira DF, Pinto E, Leite JP, Lopes AA, Oliveira-Filho J. Validation of the National Institutes of Health Stroke Scale, modified rankin scale and Barthel Index in Brazil: the role of cultural adaptation and structured interviewing. Cerebrovasc Dis. 2009;27(2):119-22. http://dx.doi. org/10.1159/000177918

8. Sablot D, Belahsen F, Vuillier F, Cassarini JF, Decavel P, Tatu L, Moulin T, Bustos EM. Predicting acute ischaemic stroke outcome using clinical and temporal thresholds. ISRN Neurol. 2011;2011:354642. http://dx.doi.org/10.5402/2011/354642

9. Furlan NE. Associação entre nível de pressão arterial e letalidade na fase aguda do acidente vascular cerebral: estudo prospectivo [thesis]. [Botucatu]: Universidade Estadual Paulista; 2015. 46 p.

10. Salat D, Campos M, Montaner J. Avances em la fisiopatologia y el tratamiento de las infecciones em la fase aguda del ictus. Med Clin (Barc). 2012;139(15):681-7. http://dx.doi.org/10.1016/j.medcli.2012.03.014

11. Soares MIFG. Acidente vascular cerebral isquêmico: complicações infecciosas segundo o volume e a localização de enfarte [thesis]. [Covilhã]: Universidade da Beira Interior; 2011. 46 p.

12. Sacco RL, Kasner SE, Broderick JP, Caplan LR, Connors JJ, Culebras A, Elkind MS, George MG, Hamdan AD, Higashida RT, Hoh BL, Janis LS, Kase CS, Kleindorfer DO, Lee JM, Moseley ME, Peterson ED, Turan TN, Valderrama AL, Vinters HV. American Heart Association Stroke Council, Council on Cardiovascular Surgery and Anesthesia, Council on Cardiovascular Radiology and Intervention, Council on Cardiovascular and Stroke Nursing, Council on Epidemiology and Prevention, Council on Peripheral Vascular Disease, Council on Nutrition, Physical Activity and Metabolism. An updated definition of stroke for the 21st century: a statement for healthcare professionals from the American Heart Association/American Stroke Association. Stroke. 2013;44(7):2064-89. http://dx.doi.org/10.1161/ STR.0b013e318296aeca

13. Centers for Disease Control and Prevention. Surveillance Definitions for Specific Types of infections [Internet]. Atlanta [USA]:CDC; 2016 [cited 2016 Mar 26]. Available from: http://www.cdc.gov/nhsn/pdfs/pscmanual/17pscnosinfdef_current.pdf

14. São Paulo. Secretaria do Estado de Saúde. Manual de orientações e critérios diagnósticos: hospital geral [Internet]. São Paulo (SP): CVE; 2016 [cited 2016 Mar 26]. Available from: http://www.cve.saude.sp.gov.br/htm/ih/pdf/IH16_Manual_Criterios_Diag_hospitalgeral_2.pdf

15. Kasner SE, Moss HE. Cerebrovascular disorders. ACP Medicine. 2010;1-22.

16. Santos ISO. Acidente vascular verebral isquêmico: fatores preditores de mortalidade hospitalar e incapacidade [thesis]. [São Paulo]: Instituto Dante Pazzanese de Cardiologia, Universidade de São Paulo; 2013. 94 p.

17. Ng YS, Stein J, Ning M, Black-Schaffer RM. Comparison of clinical characteristics and functional outcomes of ischemic stroke in different vascular territories. Stroke. 2007;38(8):2309-14. http://dx.doi.org/10.1161/STROKEAHA.106.475483

18. Muller M. Recorrência e letalidade do acidente vascular cerebral em Joinville, Brasil: estudo prospectivo de base populacional [thesis]. [Joinville]: Universidade da Região de Joinville; 2015. 103 p.

19. Pinto PTC. Trombólise intravenosa para acidente vascular cerebral isquêmico agudo em um hospital brasileiro, público e acadêmico: caracterização de casuística [thesis]. [Ribeirão Preto]: Universidade de São Paulo; 2013. 84 p.

20. Nascimento KG. Aspectos epidemiológicos e clínicos de pacientes com acidente vascular cerebral isquêmico submetido ao tratamento endovenoso com ativador tecidual de plasminogênio humano recombinante - RTPa [thesis]. [Uberaba]: Universidade Federal do Triângulo Mineiro; 2015. 80 p.

21. Westendorp WF, Nederkoorn PJ, Vermeij JD, Dijkgraaf MG, van de Beek D. Post-stroke infection: a systematic review and meta-analysis BMC Neurol. 2011(Sept 20);11:110. http://dx.doi.org/10.1186/1471-2377-11-110

22. Ionita CC, Siddiqui AH, Levy EI, Hopkins N, Snyder KV, Gibbons KJ. Acute Ischemic Stroke and Infections. J Stroke Cerebrovasc Dis. 2011;20(1):1-9. http://dx.doi.org/10.1016/j.jstrokecerebrovasdis.2009.09.011

23. Vermeij FH, Scholte OP, Reimer WJ, de Man P, Van Oostenbrugge RJ, Franke CL, de Jong G, de Jong G, de Kort PL, Dippel DW, Netherlands Stroke Survey Investigators. Stroke-associated infection is an independent risk factor for poor outcome after acute ischemic stroke: data from the Netherlands Stroke Survey. Cerebrovasc Dis. 2009;27(5):465-71. http://dx.doi.org/10.1159/000210093

24. Sellars C, Bowie L, Bagg J, Sweeney MP, Miller H, Tilston J, Langhorne P, Scott DJ. Risk factors for chest infection in acute stroke: a prospective cohort study. Stroke. 2007;38(8):2284-91. http://dx.doi.org/10.1161/STROKEAHA.106.478156

25. Cornesoltas Suarez L, Serra Valdés MA, O'Farrill Lazo R. Factores de riesgo y mortalidad por neumonía intrahospitalaria en la Unidad de Terapia Intensiva de Ictus. Medwave. 2013;13(2):e5637.

26. Paganini-Hill A, Lozano E, Fischberg G, Perez Barreto M, Rajamani K, Ameriso SF, Heseltine PNR, Fisher M. Infection and risk of ischemic stroke: differences among stroke subtypes. Stroke. 2003; 34(2):452-7. http://dx.doi.org/10.1161/01.STR.0000053451.28410.98

27. Dalager-Pedersen M, Søgaard M, Schønheyder HC, Nielsen H, Thomsen RW. Risk for myocardial infarction and stroke after community-acquired bacteremia: a 20-year population-based cohort study. Circulation. 2014;129(13):1387-96. http://dx.doi.org/10.1161/ CIRCULATIONAHA.113.006699

28. Johnsen SP, Svendsen ML, Ingeman A. Infection in patients with acute stroke. Open Infect Dis J. 2012;6(Suppl 1: M3):40-5. 
29. Wartenberg KE, Stoll A, Funk A, Meyer A, Schmidt JM, Berrouschot J. Infection after acute ischemic stroke: risk factors, biomarkers, and outcome. Stroke Res Treat. 2011;2011:830614. http://dx.doi.org/10.4061/2011/830614

30. Shaw AC, Goldstein DR, Montgomery RR. Age-dependent dysregulation of innate immunity. Nat Rev Immunol. 2013;13(12):875-87. http://dx.doi.org/10.1038/nri3547

31. Aarnio K, Haapaniemi E, Melkas S, Kaste M, Tatlisumak T, Putaala J. Long-term mortality after first-ever and recurrent stroke in young adults. Stroke. 2014;45(9):2670-6. http://dx.doi.org/10.1161/STROKEAHA.114.005648

32. Chandra RV, Leslie-Mazwi TM, Mehta BP, Yoo AJ, Simonsen CZ. Clinical outcome after intra-arterial stroke therapy in the very elderly: why is it so heterogeneous? Front Neurol. 2014;5:60. http://dx.doi.org/10.3389/fneur.2014.00060

33. Brogan E, Langdon C, Brookes K, Budgeon C, Blacker D. Respiratory infections in acute stroke: nasogastric tubes and immobility are stronger predictors than dysphagia. Dysphagia. 2014;29(3):340-5. http://dx.doi.org/10.1007/s00455-013-9514-5

34. Toscano M, Cecconi E, Capoluppi E, Vigano A, Bertora P, Campiglio L, Petolicchio MC, D'Elia S, Vicenzini VA, Cislaghi GM, Dipiero V. Neuroanatomical clinical and cognitive correlates of post-stroke dysphagia. Eur Neurol. 2015;74(3-4):171-7. http://dx.doi. org/10.1159/000441056

35. George AJ, Boehme AK, Siegler JE, Monlezun D, Fowler BD, Shaban A, Albright KC, Beasley M, Martin-Schild S. Hospital-acquired infection underlies poor functional outcome in patients with prolonged length of stay. ISRN Stroke. 2013;2013:312348. http://dx.doi. org $/ 10.1155 / 2013 / 312348$

36. Colbert JF, Traystman RJ, Poisson SN, Herson PS, Ginde AA. Sex-related differences in the risk of hospital-acquired sepsis and pneumonia post acute ischemic stroke. J Stroke Cerebrovascular Dis. 2016; S1052-3057(16):30125-2. http://dx.doi.org/10.1016/j jstrokecerebrovasdis.2016.06.008 